\begin{tabular}{|c|c|c|c|}
\hline & $\begin{array}{l}\text { Escola Superior } \\
\text { de Cestäoerior } \\
\text { Tecnologia } \\
\text { [ipsantarém] }\end{array}$ & 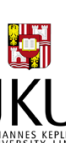 & $\begin{array}{l}\text { ISSN 2029-7564 (online) } \\
\text { SOCIALINĖS TECHNOLOGIJOS } \\
\text { SOCIAL TECHNOLOGIES } \\
2014,4(1) \text {, p. } 118-138\end{array}$ \\
\hline
\end{tabular}

\title{
CLOUD COMPUTING DEVELOPMENT IN ARMENIA
}

\author{
Vazgen Ghazaryan \\ Eurasia International University, Armenia, vazgen.ghazaryan@yahoo.com \\ Rūta Tamošiūnaitè \\ Mykolas Romeris University, Lithuania, tamosiunaite.ruta@mruni.eu \\ doi:10.13165/ST-14-4-1-08
}

\begin{abstract}
Purpose - the purpose of the research is to clarify the benefits and risks with regard to data protection and the costs to be incurred by businesses in the result of the use of these new technologies for the implementation and management of an organisation's information systems.

Design/methodology/approach - qualitative case study of the results obtained via interviews. Three research questions were raised: Q1: How can a company benefit from using Cloud Computing compared to other solutions? Q2: What possible issues occur with Cloud Computing? Q3: How would Cloud Computing change an organisation's IT infrastructure?

Findings - the calculations provided in the interview section prove financial advantages, even though the precise degree of flexibility and performance has not been assessed. Cloud Computing offers great scalability. Another benefit that Cloud Computing offers in addition to better performance and flexibility is reliable and simple backup data storage, physically distributed and so almost invulnerable to damage. Although the advantages of Cloud Computing more than compensate for the difficulties associated with it, the latter must be carefully considered. Since the cloud architecture is relatively new, so far the best guarantee against all risks it entails, from a single company's perspective, is a well-formulated service-level agreement defining the terms
\end{abstract}


of service and the shared responsibility and security roles between the client and the provider.

Research limitations/implications - the study was carried out on the basis of two companies, which gives a deeper view, but a wider analysis is necessary for the results to be applied more extensively.

\section{Practical implications:}

Originality/Value - the novelty of the research depends on the fact that the existing approaches to this problem mainly focus on the technical side of computing.

Keywords: cloud computing, infrastructure as a service (IaaS), platform as a service (PaaS), software as a service, network as a service (NaaS)

Research type: case study.

\section{Introduction}

According to Mohammad (2012), cloud computing is defined as the use of computing resources (hardware and software) that are delivered as a service over a network (typically the internet). The name comes from the use of a cloud-shaped symbol as an abstraction for the complex infrastructure it contains in system diagrams. Cloud computing entrusts remote services with a user's data, software and computation (ibid). Recently Cloud Computing has gained high importance in managerial process across various sectors. It is much discussed not only by the media but also by analysts as it offers unique opportunities for both business organisations and public institutions. Armenian information technology (hereinafter - IT) and high technology industries registered enormous growth during the previous decade. Soon it became one of the most active and internationally competitive branches of the Armenian economy. The majority of Armenian ICT companies have been trying to decrease computing costs, for that reason most of them start using virtualisation technologies. Cloud Computing may let them further reduce costs. It can improve utilisation, reduce infrastructure and administration costs. According to Michael Armbrust et al. (2009) "most of growing, starting or expanding IT intensive organizations would probably save costs and gain flexibility when using Cloud Computing." Nevertheless, it is necessary to research this assertion: firstly, to understand whether it is a veritable statement or not, and, secondly, to research if the profits of having Cloud Computing transcend the risks.

The problem appears when Cloud Computing is used in order to enlarge, start or just temporarily use this technology. Companies move their processing units to another location which modifies the infrastructure.

Qualitative research method was used for the research. We have carefully reviewed and looked at Cloud Computing scientific literature and discussed the most important details with experts. The research part contains interviews taken from two Armenian IT companies. After this we used empirical data from the scientific literature and tried to describe the risks and benefits of this technology. 
We will not consider private users. In this research, only Cloud Computing business users, i.e. companies that significantly use IT technologies will be taken into account. To be more specific, companies that have any relation to Cloud Computing or companies starting, enlarging a business or in need of some extra IT services were included in the research. In this case, matching IT companies were interviewed. Technical aspects of Cloud Computing are another important issue on the agenda, which has been covered briefly, as it falls outside the study field of this research.

Soon the traditional IT system can be replaced by Cloud Computing in Armenia, which will make IT organisations more flexible. With Cloud Computing, they can save costs and process information and data much faster than with traditional IT. In any event, we could face the problem risking this new technology. It is extremely important to realise whether the use of Cloud Computing can bring value to ICT intensive companies or not.

Various aspects of Cloud Computing must be taken into consideration. Possibilities, risks, and problems that may arise while using Cloud Computing should be pointed out in order to understand if it is profitable or useless to implement this technology for Armenian ICT intensive companies. Novelty of the research depends on the fact that existing approaches to this problem mainly focus on the technical side of computing (Harutyunyan and Grigoryan, 2010; Harutyunyan et al. 2011; Mohammad, 2012; Xu and Zhong, 2014; etc.). Moreover, these approaches are often focused on the end user of the cloud and not the companies using it.

The purpose of the research is to clarify the benefits and risks in regard to data protection, the costs that businesses can incur by using these new technologies for the implementation and management of an organisation's information systems. The objectives are to analyse Cloud Computing risks; to examine advantages of Cloud Computing; to research possible implementation of Cloud Computing. Three research questions were raised: Q1: How can a company benefit from using Cloud Computing, compared to other solutions? Q2: What are the possible issues that occur with Cloud Computing? Q3: How would Cloud Computing change an organisation's IT infrastructure?

\section{Overview of Adacemic and Practical Bases of Cloud Computing}

The word cloud is used to describe Cloud Computing because of the metaphor used to describe networks, a cloud that underlies all the technology that is above and the user is unaware and doesn't have to know of its existence. There is no formal definition of Cloud Computing. However, some important organisations and experts offer their own versions of definitions. According to the National Institute of Standards and Technology (Liu, 2011), "Cloud computing is a model for enabling ubiquitous, convenient, on-demand network access to a shared pool of configurable computing resources (e.g., networks, servers, storage, applications, and services) that can be rapidly provisioned and released with minimal management effort or service 
provider interaction". According to Mohammad (2012), Cloud Computing amounts to "Computation, software, data access, and storage services that do not require enduser knowledge of the physical location and configuration of the system that delivers the services". Armbrust et al. (2009) refer to "both the applications delivered as services over the Internet and the hardware and systems software in the datacenters that provide those services." Definitions by a number of other authors could be presented, though the concept is not that contradictory so that to deserve more attention. In general, Cloud Computing is a paradigm based on the consumption of resources, applications, hardware or computation, offered by the internet and consumed on demand. To illustrate this, Jonson (2013) presented a visual model of Cloud Computing (see Figure 1).

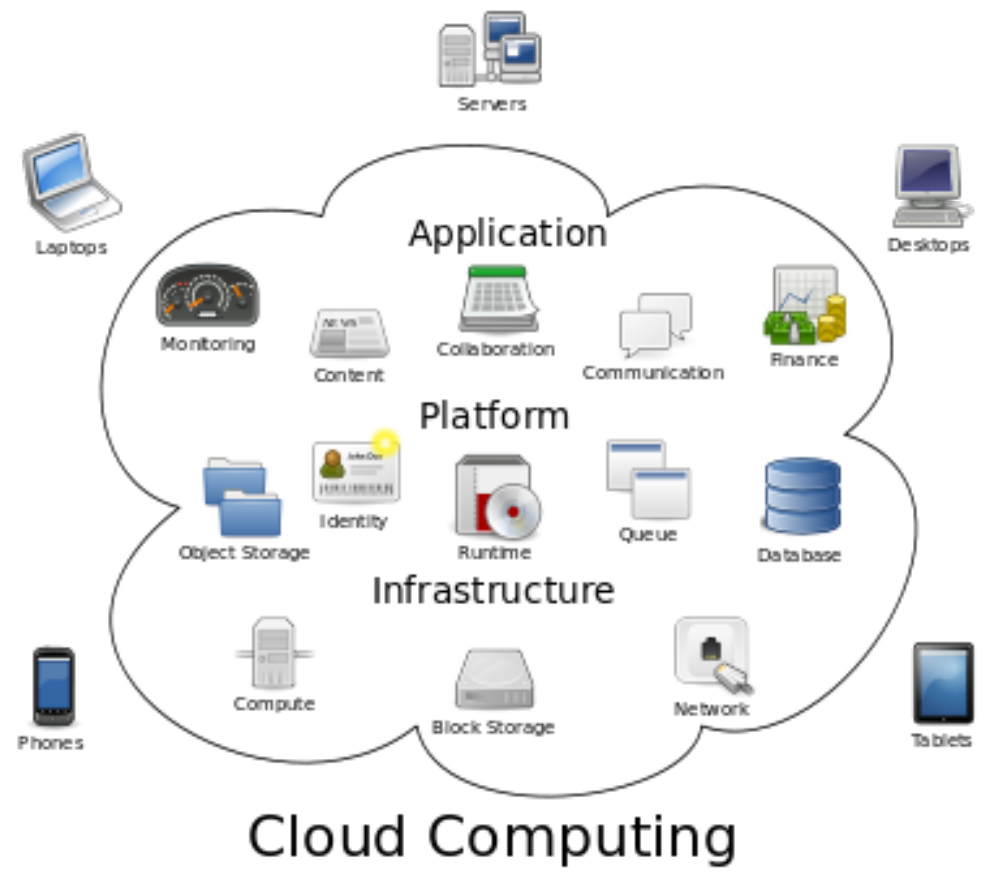

Figure 1. Visual model of Cloud Computing (Johnston, 2013)

The National Institute of Standards and Technology (NIST) of the U.S. Department of Commerce defines Cloud Computing architecture by describing five essential characteristics, three cloud services models and four cloud deployment models (Liu, 2011) (see Figure 2). The five major participating actors in the process are Cloud Consumer, Cloud Provider, Cloud Broker, Cloud Auditor and Cloud Carrier. The essential characteristics of Cloud Computing which explain the relations and the difference from traditional computing are as follows:

On-demand-self-service. The supply of such computer services as applications, e-mail, network and server services is carried out without human cooperation with each service provider. For instance, Amazon Web Services (AWS), Microsoft, Google and Salesforce.com. 
Broad Network Access. Cloud Capabilities can be obtained through the network and accessed through standard mechanisms that promote use by heterogeneous thin or thick client platforms, such as laptops, mobile phones and PDAs.

Resource Pooling. Computing resources of providers are linked to each other. In this way, they serve different clients who use various-tenant model, with different physical and virtual stocks dynamically reassigned and appointed. In this stocks processing, memory, virtual machines, network bandwidth and email services are contained. Resource linking with each other serves as a ground for scale economies.

Rapid Elasticity. Cloud service can be supplied quickly and flexibly, even automatically in some situations to scale out and quickly liberated to fast scale in. For the customer, the potentials reachable for supplying frequently appear to be limitless and may be bought at any time and in any amount.

Measured Service. Cloud computing resource usage can be controlled, measured, and reported providing transparency for both the consumer of the utilised service and cloud provider. Metering can be used by cloud computing services to control and optimise resource use.

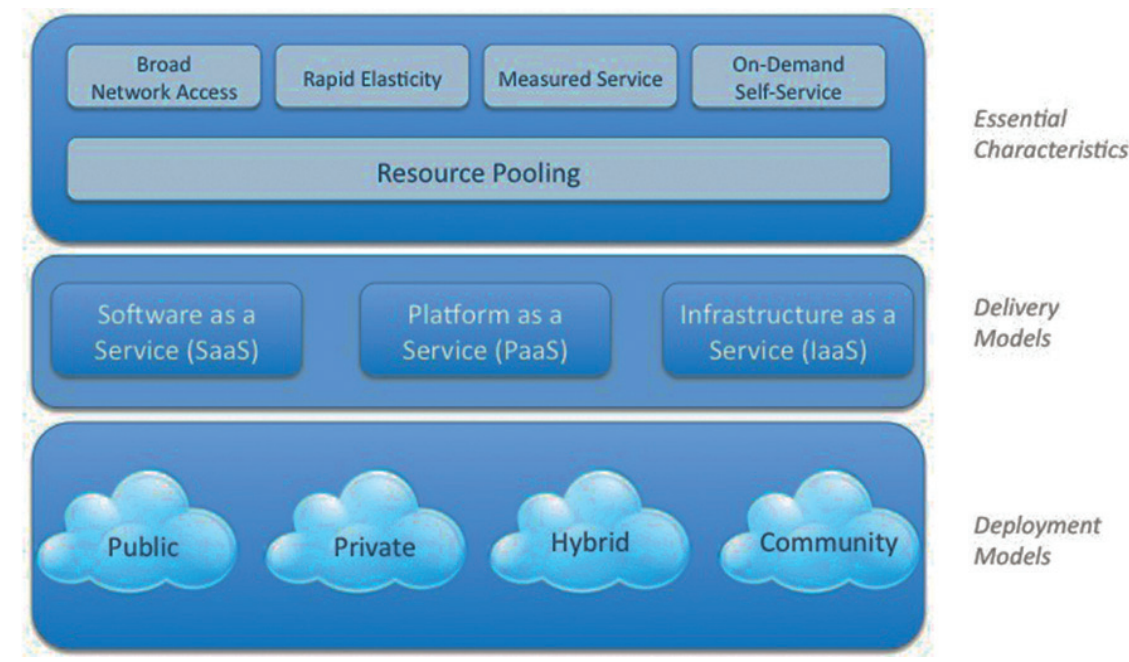

Figure 2. Visual model of Working Cloud Computing Structure (Liu, 2011)

Cloud Services Models and these three fundamental classifications are often referred to as SPI models (Software as a Service, Platform as a Service and Infrastructure as a Service.)

- Cloud Software as a Service (SaaS). The customer is provided with availability. That is the use of provider's requests persisting a cloud infrastructure. The requests may be attainable from different customer machines via a thin customer interface, e.g., a web browser. The customer does not control the beneath cloud interface. These include servers, a storehouse, network servers and even private request availabilities, with the probable exclusion of form settings of restricted user-specific application. 
- Cloud Platform as a Service (PaaS). The customer has the right to spread out client-created or obtained applications onto the cloud infrastructure in order to use programming languages and devices supported by the provider. The customer doesn't control the beneath cloud interface, involving network, servers, acting systems, or storehouse, excluding probable requests hosting, environment forms and spread out applications.

- Cloud Infrastructure as a Service (IaaS). The customer has the right to purchase processing, a storehouse, networks and other kinds of basic computing stocks. In it a customer may spread out and move arbitrary software that can involve working systems and applications. The client does not manage the beneath cloud interface but has access to the operating system, the storehouse, spread out applications and probable limited management of networking elements.

- Cloud Deployment Models (CDM)

- Private cloud. The cloud is operated only for a company. The company or a third party may control it. It can subsist on condition or off condition.

- Community cloud. Some deferent companies are sharing the cloud infrastructure. It encourages communities which have shared affairs such as protection demands, strategy, etc. The company or a third party may control it. It can subsist on condition or off condition.

- Public cloud. The common public and industry may have access to the cloud computing. The cloud computing belongs to a company selling cloud services.

- Hybrid cloud. The cloud interface consists of two or more clouds. These clouds remain unique entities but at the same time are linked to each other in a standard way. Thereby they empower data and application portability.

Current structure of Cloud Computing was developed gradually. The history of computing goes back to the invention of the calculating machine. Wilhelm Schikard was the first person to document the collection of such a calculating machine in 1623 . Another period of computing history is the Analytical Engine description made by Charles Babbage in 1837. It is a calculating machine for special purpose tasks. Extra periods were Allan Marquand's draft of an electrical logic machine in 1885 and Herman Hollerith's development of a tabulating machine in 1890. Benjamin Burack was the first man to produce an electrical logic machine in 1936. The modern computer history starts in 1941 with Konrad Zuse's construction of Z3. That machine was the first functioning digital computer which was based on the binary digit system, Turing capable and programmable. The first electronic tube computer was the Electronic Numerical Integrator and Computer (ENIAC) which was built by John Mauchley and J. Presper Eckert in 1945. The ENIAC was based on the decimal system, but was both programmable and Turing capable.

Future growth of technologies like Java, PHP/MySQL or Ajax and increasing bandwidth made it possible to develop better websites. Owing to this development, now we can find numerous multimedia websites (Flash and Silverlight), many webbased applications and online shops that are spread out through the Internet. Route planners, social networks, communication platforms and also total office applications (word processors, spread sheet applications) are bright examples. In 2000 this 
concept became famous and it was sometimes referred to as Software-as-a-Service. Similar deployment forms were developed for the deployment of hardware resources, particularly computing power and storage. Only in 2007 the term Cloud Computing appeared. It typically represents joint hardware and software deployment concept. Google and IBM together with six American universities began researching cloud technologies.

Looking back at the 1960s and 1970s we may see that the operating models of that period are coming back with Cloud Computing. These old trends are centralised by shared computing resources. Time-sharing concept traces back to John McCarthy in 1957. In this concept, idle CPU times where dynamically distributed to several users. In the past, computers, data processing and also their operation was costly and that is why the operators tried to find ways to use it in the most beneficial way. Thus, those companies that had mainframe computers offered their computing output to external users. It served as a basis for further establishment of independent service providers specialising in the deployment of computing resources. The Service Bureau Corporation or Tymshare Inc (IBM's subsidiary), General Electric's Information Service Company (GEISCO) are the examples of those companies that offered timesharing systems. In 1990s a new trend appeared and it was created to continue with Cloud Computing. With that trend we go back to the period of centralisation of information technology, centralisation of data storage in particular. Amazon, Google and other modern datacentre operators opposed this situation. The same happened in the 1960s and 1970s. They also were trying to use their huge output in a better way. Virtualisation and other modern approaches are effective ways to confer third party user access to their infrastructure and harness computing power and storehouse abilities.

The Australian Information Industry Association (KPMG, 2012) modelled the economic impact of Cloud Computing and grouped the advantages into three broad categories:

1. Direct cost savings (decreased price per production unit) - the largest and the most recognisable economic profit of Cloud Computing is direct cost savings from changes inside the organisation and outside scale economies.

2. Productivity improvements (increased production cost unit) - in order to change something in business, there is no need to plan the capacity in details, established technology changes or new technology acquisition.

3. Innovation (capability to deliver new and developed output) - additional profits that can be gained by organisations are quickness, flexibility, cooperation and presenting new output and services on the market.

The largest and the most recognisable economic profit of Cloud Computing is direct cost savings. Direct cost savings for organisations derive from the changes inside the organisation as well as data centres that contain the IT infrastructure. "Direct cost savings occur at the data centers through significant economies of scale in three areas." (Microsoft, 2010).

The accomplishment of Cloud Computing can bring changes into business without detailed planning of capacity and it can also modify the installed technology or new 
technology acquisitions. As a business product, it permits the geographical removal of office openings, stuff and operations out of business systems' compromising access. It also allows implementing an idea and satisfying new business demands much faster than before.

Organisations are empowered by cloud to go up and down to the demanded service level which led to optimise demanded capacity and decreased prices. The on-demand up/down flexibility of cloud-based computing services let the capacity to rapidly raise computing stocks to select business increase while minimising risks downside, that is to say, protecting the capability to free resources in the case of failure of a programme to get support.

Moreover, Cloud Computing let personnel have access to data and files in the case they are working at a distance or outside their working hours. E-commuting may generate large potential profits to businesses (by reducing overheads) and to users.

Cloud Computing can be especially useful for small companies. There can be shortage of capital to obtain the in-house ICT methods of solutions demanded in the case of no cloud service. The profits of Cloud Computing can also transfer into 'time to market' for client-facing activity that is much faster. There are great potentials to establish new services and to adapt already existing ones faster in response to feedback or changing client demands. For some situations this would mean that the developments are obvious, scaling down from months to weeks and from weeks to days. Companies can either make progress in new service building and output on cloud platforms taking entire advantage of centralised data, simple scalability and web accessibility.

Risk should always be understood in relation to general business chance and tend to risk - from time to time risk is rewarded by opportunity. The European Network and Information Security Agency (ENISA) grouped Cloud Computing risks into three categories (Cloud Computing Risk Assessment, 2009): policy and organisational (lock-in, loss of governance, compliance challenges, loss of business reputation due to co-tenant activities, cloud service termination or failure, cloud provider acquisition, supply chain failure); technical (resource exhaustion, isolation failure, cloud provider malicious insider - abuse of high privilege roles, management interface compromise, intercepting data in transit, data leakage on up/download, intra-cloud, insecure or ineffective deletion of data, distributed denial of service (DDoS), economic denial of service (EDOS), loss of encryption keys, undertaking malicious probes or scans, compromise service engine, conflicts between customer hardening procedures and cloud environment); legal (subpoena and e-discovery, risk from changes of jurisdiction, data protection risks, licensing risks). Some risks can affect Cloud Computing, but no risks specific to it were revealed as well: network breaks, network management (i.e. network congestion/mis-connection/non-optimal use), modifying network traffic, privilege escalation, social engineering attacks (i.e. impersonation), loss or compromise of operational logs, loss or compromise of security logs (manipulation of forensic investigation), backups lost, stolen, unauthorised access to premises (including physical access to machines and other facilities), theft of computer equipment, natural disasters. 


\section{Cloud Computing in Armenia}

Looking back at the history of Armenia we can notice that Armenia was in one of the leading positions in high-tech development and manufacturing. During the Soviet period, Armenia was the main figure of USSR's critical scientific and R\&D activities in many technological industry branches, e.g., mainframe and industrial computing, semiconductors, software development, electronics, etc. Armenian technology branch was mainly focused on extensive R\&D and production projects which mainly cooperated with industrial and military applications. This was the situation before the collapse of the Soviet Union. After its collapse, after regaining independence in 1991, software development, IT services and outsourcing was in the spotlight.

During the last ten years, Armenian IT industry expanded and grew rapidly. Soon it became one of the most flexible and internationally competitive branches of Armenian economy.

The Armenian Government acknowledged the fact that there was a need to establish a full legislative, regulatory and institutional framework as an important condition after an official declaration in 2000 identifying the country's IT as a strategic priority. For fulfilling this task, the Armenian Government developed a sequence of important stages, starting from the adoption of IT-related legislation to becoming a signatory to several conventions and treaties belonging to global information and communication technologies standards.

In 2001, as a member of this obligation created for steady IT development, the Armenian Government developed an IT Strategy and an IT Implementation plan to encourage IT and present Armenia as an important IT centre of the region. In May 2001, the Ministry of Trade and Economic Development of the Republic of Armenia prepared an IT Development Concept Paper and an Action Plan that was adopted the same year. Information Technologies Development Support Council of Armenia (ITDSC) was founded by a third component foreseen in the 2001 Presidential Decree, then presided by the country's Prime Minister, and involving some other pertinent stakeholders and parties. 2002 saw the creation of the Enterprise Incubator Foundation. It was the posterior institutional stride forward.

The Armenian Parliament in its turn adopted some essential legislative initiatives and reforms intended to support IT branch development. All this process began in 2000, when the Parliament of Armenia adopted the Law on Scientific-Technical Activity. It provided a structure for state R\&D efforts. In 2003, supplementary laws were adopted. Among these were the Law on Freedom of Information and the Law on Public Notifications. The first one provides underdeveloped structure for public access to governmental information, legislation and decisions, and the second one manages the terms and methods of public notices via the internet and contains e-government programmes, such as web-based procurement.

In 2004, two important laws were passed by the Armenian Parliament. The first one was the Law on Archival Operations. It was intended to control the collection and storage of information. However, regulation should aim for ultimate privacy security covering medical records and some private data. The second one adopted was the Law 
on E-Digital Signature. It regulates all aspects related to e-documents and supervises the form and use of electronic finger-shaped signatures in the sphere of electronic commerce. During the following two years, the Parliament adopted the 2005 Law on E-Communication, amending the Criminal Code to involve computer crimes. In 2006, another law was adopted, namely the Law on State Support to Innovation Activities. This one is aimed at serving as a legislative basement for the country's Innovation System Development Programme. The latter is a plan to create a national innovation system via building institutions in the scientific-technical and industrial-technological districts, to create risky and innovation reserves, techno-parks, business incubators, and to promote scientific and educational institutions involved in technology transmit.

The 2012 Armenian information and communications technology sector state of industry report (2013) emphasised that: "Currently, the Armenian Parliament is considering a draft Law on Information Technologies, Information and Information Security, a move that promises to further prepare the foundation for a more ambitious and extensive effort of IT development."

In North America and EU countries, the use of IT is much more developed than in Armenia, which is why few companies use Cloud Computing infrastructure. Software and service industry in Armenia is rather young but it is growing very quickly. And in the future many companies will use Cloud Computing technologies.

\section{3. "Massaca LLC” and "Ayb Host LLC" Case Analysis}

The following two companies were interviewed: Massaca LLC and AYB Host LLC. The data collected with interviews is instantly aligned with literature. IT companies chosen for interviewing were expected to be innovative and proactive in development. Massaca is not a large web development company and at present they are working on a web-based CRM tool. Their essential business is based on IT usage. Moreover, their outputs need IT for operation. Of course, now they are a small company, but the perspectives for development are evident. The second company, AYB Host LLC is a software development company. It is located in the capital of Armenia, Yerevan. This company is also an IT organisation and it used to manifest their fundamental business activity.

\subsection{Massaca LLC (Web Development Company)}

Massaca LLC is a web development company. It was founded in 2008 in Yerevan, Armenia. At present, CRM tools are provided by the company and used by their clients to control the cooperation of the company with current and future customers. These tools may be viewed as portals placed on the internet. The created solutions are particularly designed for the Armenian market, intended for small and medium-sized businesses and accessible for a small monthly fee.

Current use of IT infrastructure. This company has faced some IT interface change in March 2013. A few years ago, they had a data centre in their current office. In a 
reserved small room, they had two servers for two purposes; one to run the application in their office, and the other one for hosting their websites. Soon they decided to modify their IT infrastructure, as the servers were too noisy. Therefore, the servers were placed at a third party data centre in Armenia. The first server is for hosting all their data and software for ordinary routine operations and the second one is for hosting their websites. It is an essential growth factor that they want to change the use of telephone lines to VoIP. It is rather difficult to fulfil this task, as several lines are often needed for VoIP. The use of VoIP should be much more profitable than the use of traditional lines. In their everyday affairs, they use different software packages (open-sourced or paid software). They also use Microsoft Word, Excel, PowerPoint, Microsoft Publisher, etc. included in the Microsoft Office package.

Current security handling. Anti-virus and firewall are the most ordinary means to guarantee their security. For PC users, this software is enough, but a company like this needs much more. There are many security demands to be satisfied in order to guarantee a certain level of protection. The fact that there is a connection between their office and the servers causes a security risk. As their services are mostly concentrated on the Armenian market, they use IP blockade in their servers. Thus, it follows that if someone tries to hack others with IP addresses of foreign countries, they cannot access servers as the server does not allow these IP addresses to access servers. The software that is used to realise remote desktop connection also protects the link between the office and the server. Remote desktop connection has a built-in protection measure that doesn't allow connecting to the server without a username and a password.

Current issues. One of the main issues is that they have to contract out their servers, which means that they are no more responsible for their keeping and realising. They contract company data out into servers that belong to server providers. Despite the fact that they have service contracts with these providers, they could face rather serious problems in case of server fall for some period of time. In this situation, Massaca is not in charge to change anything. Thus, the only thing that needs to be done is mentioned in their service contract. Company growth may also bring some problems. Buying new hardware is very easy, but the problem is to transfer this much of data, as the server is overloaded. To solve this problem, you will need more servers to withstand this data increase. This very process may be not effectual and it may lose much time, as there will be a need to buy some new server and the data will need to be integrated into this server. Some employees keep their passwords on their PCs or laptops which makes their password unprotected and easy to break. And the result is that third parties can have access to their critical data and own their passwords. Therefore, we conclude that the entire chain is as strong as the weakest link. Here we have the human as the weakest link. All security means are meaningless, if an employee does not use his password carefully.

Benefits. From the point of view of Massaca, it can be rather profitable to add some new software, hardware and even the entire traditional IT infrastructure. They think that the use of Cloud Computing and possibly training or employees may increase costs. Therefore, they do not expect any price advantages from Cloud Computing. We have tried to apply the literature to the case study of Massaca in order to conclude 
something useful about the benefits of Cloud Computing, compared to traditional IT infrastructure which they currently use. "The largest and the most identifiable economic benefit of cloud computing is direct cost savings. Direct cost savings for organisations occur from changes both within the organisation, and also large data centres housing IT infrastructure." (KPMG, 2012). Comparing Massaca's current IT expenses with the same Cloud Computing solutions (Tables 1.4 and 1.5), we can see that Cloud Computing reduces costs by almost $40 \%$ a month.

This company uses several software packages to keep office. Thus, the use of Cloud Computing may bring many benefits for this company. They can use Software as a Service (SaaS) solution to run their applications. "Cloud Software as Service (SaaS): The capability provided to the consumer is to use the provider's applications running on a cloud infrastructure. The applications are accessible from various client devices through a thin client interface such as a web browser. The consumer does not manage or control the underlying cloud infrastructure including network, servers, operating systems, storage, or even individual application capabilities, with the possible exception of limited user-specific application configuration settings." (Liu, 2011)

Therefore, they do not have to install software on their office computers or servers. It will remove expect, installation problems and support. When the customer wants to buy the right to use these parts of software, he/she pays the real price for the licenses. One can get software whenever they want, because the system of Cloud Computing is very flexible. As an example, when a company needs one software only for one project, it can buy access rights for several weeks only. It is very useful not to have to buy the entire software license.

The use of cloud as an infrastructure (IaaS) is another step forward. "Cloud Infrastructure as a Service (IaaS): The capability provided to the consumer is to provision processing, storage, networks, and other fundamental computing resources where the consumer is able to deploy and run arbitrary software, which can include operating systems and applications. The consumer does not manage or control the underlying cloud infrastructure but has control over operating systems, storage, deployed applications, and possibly limited control of select networking components (e.g., host firewalls)." (Liu, 2011). Recently, Massaca has removed all of its private servers from the office to a server provider. Therefore, it would amount to transferring their servers into the cloud with advantages of SaaS usage for their software.

Issues. At present, they have outsourced their servers to one Armenian data centre that uses traditional IT infrastructure. From here we can suggest that they have their central data running via a third party. This is more expensive, compared to Infrastructure as a Service (IaaS), which acts as an internal server provider. There are some differences between them as well, such as the fact that traditional service provider is not as reliable as Cloud Computing, even if they obtain a good service contract (SLA). Moreover, Cloud Computing has more reserved capabilities and stocks in the cloud itself than a traditional service provider has on its private servers. The most basic thing about the IaaS is its ability to scale dynamically very easily in relation to the application resources needs. It suggests that whenever a server has operational problems, it can be changed by other servers easily, so that cloud customers do not face stoppage. In 
traditional IT infrastructure, new obtained servers have to be connected and combined with the existing ones and the data needs to be integrated. It follows from this that it is not very suitable to adapt new servers to the rapidly lifting data remove and customer. In the case of a contract mentioning the use of Infrastructure as a Service (IaaS) there is a constant dynamically available infrastructure. The infrastructure referred above can be enlarged very easily. Thereby, the lifting quantity of customers may use more data in the application.

Security. At present, they use antivirus and firewall software on a moderate level and customers should be protected all the time using this software. A cloud can also apply this software, depending on the services it provides. Currently the access to their servers is restricted for foreign IP addresses and it is impossible to connect to servers from outside of Armenia. As referred above, they mainly work for the Armenian market. Thus, this fact does not guarantee that it will not be attacked from Armenia. For someone in Armenia (competitors, for example) it could be more profitable to attack than for a foreigner. The Cloud Computing provider secures the connection between customers and the cloud. Particular protection of these connections is defined by Service Level Agreement (SLA). For various employees, they try to share rights in the remote servers. One can ascribe to a group of users who are pre-specified by Cloud Computing. And in this case it will not be necessary to define every new user.

Cost analysis. Expenditures incurred on current IT infrastructure are valued at AMD 8000000 AMD (about USD 20 000) per year. Let us divide these prices into several groups and then return to monthly costs. AMD 4000000 goes to equipment and maintenance, AMD 3000000 to licenses and finally AMD 1000000 is allocated for support.

Table 1. Current IT expenses of Massaca LLC

\begin{tabular}{|l|c|c|}
\cline { 2 - 3 } \multicolumn{1}{c|}{} & Yearly IT cost (AMD) & Monthly IT cost (AMD) \\
\hline Equipment & 3000000 & 250000 \\
\hline Maintenance & 1000000 & 83333.33 \\
\hline Licenses & 3000000 & 250000 \\
\hline Support & 1000000 & 83333.33 \\
\hline TOTAL & $\mathbf{8 0 0 0 0 0 0}$ & $\mathbf{6 6 6 6 6 6 . 6 6}$ \\
\hline
\end{tabular}

Equipment: Two dedicated servers. The first server is for hosting all their data and software for ordinary routine and the second one for hosting their websites.

Maintenance for all equipment they use.

Licenses: They use several software packages (Operational Systems, Microsoft Office and other specific software), and they pay for these packages a yearly fixed amount.

Support for servers.

In Yerevan, Armenia we found a local Cloud Computing provider that perfectly suits this company.

24 GB RAM and 2TB disk space for server is rather sufficient for their central business. Monthly cost of this service would be around AMD 150 000. They will need 
approximately AMD 200000 per month for equipment, maintenance and assistance which are essential. Resources for the systems are also essential. Cloud provider's offer is 250 GB backup servers at AMD 50000 per month.

Table 2. Benefits of using Cloud Computing for Massaca LLC

\begin{tabular}{|l|c|c|}
\cline { 2 - 3 } \multicolumn{1}{c|}{} & Yearly IT cost (AMD) & Monthly IT cost (AMD) \\
\hline Server & 1800000 & 150000 \\
\hline Equipment, Maintenance and Support & 2400000 & 200000 \\
\hline Backups & 600000 & 50000 \\
\hline TOTAL & $\mathbf{4 8 0 0 0 0 0}$ & $\mathbf{4 0 0 0 0 0}$ \\
\hline
\end{tabular}

As can be seen from the table above, the level of Cloud Computing differs importantly, compared to the present prices. Cloud Computing cuts back on expenditure about $40 \%$ a month and thereby it provides more elasticity for companies.

\section{Case conclusion}

We can conclude that Massaca will benefit from using Cloud Computing. They will gain profit by using Cloud Computing. Thus, let us look at the costs of Massaca incurred for their current IT infrastructure. If we compare it with the prices of Cloud Computing, we can clearly see that the latter take priority; AMD 8000000 AMD 4800 000. The system they are using now can be operated with the use of SaaS/ IaaS very easily. In addition, there are several similarities between their current system and Cloud Computing, because they have remote server to connect to.

SaaS, which provides better service and assistance capacity and is more flexible, can help this company solve some problems that it is facing now very easily. Despite the fact that Cloud Computing provides more profit, compared to their current system, and the risks are smaller, in this case this company would not like to present this new system in one platform. First of all, Cloud Computing will be used by them for the particular parts of their business. And this usage will be extra. In the future, it will be obvious whether Cloud Computing is as good in practice as in theory, and only after that they may solve more serious problems in the cloud.

\subsection{AYB Host LLC (Software Development Company)}

AYB Host is a prominent innovative software development company founded in Yerevan, Armenia in 2002. Their software and IT are aimed at helping other companies in their activities. AYB Host has two offices and a large number of customers and contacts not only from Armenia but also from abroad. For them, creativity in this field means being able to cooperate with modern technologies and always suggest innovation solutions.

Current use of IT. They use diverse forms of IT hardware and software. First are PCs, consisting of laptop and desktop computers. Both laptop and desktop computers have to be connected to a network cable. For them it is rather risky and dangerous to 
have a wireless network. In addition, they do not see any profits and advantages from using wireless networks for their everyday routines. They do not use Cloud Computing themselves in their offices for fulfilling everyday routines. All their IT is controlled via in-house servers and one remote server outsourced to a third party data centre. All the tools that are offered by this company to their clients are licensed and they also buy all the necessary licenses that are essential to be able to develop software for end users. In their office, Microsoft Office package is mainly used, particularly MS Word and Excel. They also use web-based financial application and other online tools. Basically, we can notice little usage of Cloud Computing (SaaS) in their business. Besides these software packages, they have a Customer Relationship Management (CRM) tool carrying on their servers. One of the most important things to mention is their Intranet SharePoint. They use SharePoint as a development environment. It makes it easier to develop software for customers. All the time they need to buy software or hardware and first of all they reckon up and try to select the best version. Afterwards they have to understand and decide whether to accomplish or add it to their servers, or contract it out to a third party data centre.

Current security handling. This company is responsible for the security of its own data and applications and various protection methods are used. Nearly all software and hardware they use is their own, not obtained from Cloud Computing. Let us start from their own network domain security. The central server uses Windows as an operating system and connects to particular group of computers. Those sectors make it possible to group the users who are the only ones having access to the account and password combination. This helps sharing various rights between different groups and different users. This means that rights can only be classified in a sector not to be restricted user by user. It is worth noting that they do not maintain the servers themselves and leave it to third parties, as AYB Host does not have enough knowledge and time for this. In addition, productivity will decline if employees try to support the server.

The fact that there is no wireless network to access the servers, in combination with physical security makes it more difficult for other persons to attack the servers from outside. They provide their protection by using ID entrance cards and secured doors that can only be opened from inside and by the staff only. It should be kept in mind that, in addition to the means employed for the protection of the most important things are employee alertness and awareness. And as a company they try to keep their employees informed about the security risk.

This company uses Kaspersky Antivirus and firewall software to protect PCs. These will stave off gaps from the internet. The good thing about them is that they only want to use licensed software. If something fails in software, the provider of this software would be responsible for the failure. Thus, for all of the above-mentioned reasons they forbid the use of non-genuine software in their company. They also try not to use open source software as then hackers may access scripts very easily.

Implementation issues. Currently they have had some different projects using Azure platform. The Microsoft Azure is hosted on their remote server. Now they want to use Azure in a Cloud Computing environment (PaaS). The main question here is the lack of knowledge. As Cloud Computing is quite new and has no widespread 
usage yet, there are many unknown factors. By the way, Cloud Computing has not been extensively used not only in Armenia but also abroad. Primarily they should define what they can and cannot do with it. Somehow it can turn to the process of fault and challenge. They also cultivate methods that were changed during time. It can be possible to connect a new platform with the current one and check them with the use of Cloud Computing in a different way. However, this is also an issue of knowledge.

Another problem that can arise is related to the lack of trust in the Cloud Computing platform. AYB Host trusts in Cloud Computing, but it may lack partner confidence. They have some joint projects with partners going on and it is rather difficult to convince a company to use Cloud Computing as it is quite new and we cannot see too many examples of its successful application. This makes the IT situation very difficult.

As for them, they do not know about the assistance that can be drawn from the Azure platform and they are also unaware of what will happen when something goes wrong with the Cloud Azure platform as they have not faced this yet.

Risks and benefits. With the use of Cloud Computing, we can definitely encounter both profits and risks. Among the risks, AYB Host first mentioned the fact that one could give up managing their IT system. In the case of in-house system, one can just walk up the physical systems and test everything. With Cloud Computing it is a bit different as one depends on the service provider and at the same time it becomes more difficult to supervise scalability. With Cloud Computing one may enlarge computing power where necessary. Now the benefits should be mentioned. The important benefits of using Cloud Computing are the pay-as-you-go principle, scalability and standardisation. Having reserved resources for peak IT usage is unnecessary with the pay-as-you-go system. There are only changeable prices with peak usage and no fixed ones. The scalability that Cloud Computing provides is also very important. Referring to standardisation, it makes handling systems and altering installation easier.

Benefits. AYB Host uses Microsoft Azure on a remote server to develop applications for their customers. Remote servers have some similarity with Cloud Computing. Thus, they are already familiar with this system and somehow have confidence in this new technology. In the case of using Cloud Computing, their in-house and remote servers by which all their IT is controlled can be migrated to Infrastructure as a Service (IaaS) platform. All types of computing resources, such as networks, storage, servers can be supplied by Cloud Computing. All the other office applications that are used by them at present and obtained via licenses can be transferred to the Software as a Service (SaaS) platform. More essential is that company's Customer Relationship Management (CRM) also could run in the cloud environment. Every time new hardware or software is necessary they decide to take it in-house or to contract it out. In the cloud, this can be better and easier accomplished. This has its benefits; Cloud Computing is much more flexible than in-house IT and it is also cheaper than outsourcing to a third party (traditional IT infrastructure).

Issues. Some issues are inevitable while using Cloud Computing. The first relates to the lack of knowledge and afterwards the problem of confidence in the technology arises. Another problem is that AYB Host partners have to withstand two different 
systems with different storehouses and servers. It would be rather difficult to connect these two parts of IT.

Security. Now they have Windows server that only provides access to predestined computers. Cloud Computing offers a much more beneficial solution. It provides easily groupable users with their own passwords and usernames. The support of their IT systems is contracted out, while in the case of Cloud Computing the support is already in the price of using Cloud Computing. The cloud provider withstands it. There is no representative for physical protection offered by Cloud Computing. Cloud access can be organised in a way that one can have access to it only at particular times. Physical protection of Cloud Computing need not pay that much attention to hacking. Cloud providers always use genuine software. That is why it can never be encumbered with illegitimate software copies. Moreover, the cloud provides software that is not open source. This fact expels purchasing of open source software for cloud users. Finally, Antivirus or Firewall software can be provided by the cloud for the customer PCs.

Costs analysis. AYB Host regards Cloud Computing as more supplementary piece of IT and sees it as a tool that will give rise to income. They do not want to speak about current expenses on IT infrastructure. It is an average initial small IT company that will use Azure for their IT in contrast with traditional IT.

Table 3. Azure with traditional IT

\begin{tabular}{|l|c|c|}
\cline { 2 - 3 } \multicolumn{1}{c|}{} & Yearly IT cost (AMD) & Monthly IT cost (AMD) \\
\hline Equipment & 4800000 & 400000 \\
\hline Licenses & 2400000 & 200000 \\
\hline Support & 960000 & 80000 \\
\hline Maintenance & 960000 & 80000 \\
\hline TOTAL & $\mathbf{9 1 2 0 0 0 0}$ & $\mathbf{7 6 0 0 0 0}$ \\
\hline
\end{tabular}

Equipment: Servers.

Licenses: All the software used.

Support for licenses and equipment.

Maintenance: equipment.

The same comparison can be made with Cloud Azure (PaaS).

Table 4. Azure with Cloud Computing

\begin{tabular}{|l|c|c|}
\cline { 2 - 3 } \multicolumn{1}{c|}{} & Yearly IT cost (AMD) & Monthly IT cost (AMD) \\
\hline Equipment & 2880000 & 240000 \\
\hline Licenses & 480000 & 40000 \\
\hline Support & 480000 & 40000 \\
\hline Maintenance & 240000 & 20000 \\
\hline TOTAL & $\mathbf{4 0 8 0 0 0 0}$ & $\mathbf{3 4 0 0 0 0}$ \\
\hline
\end{tabular}

Equipment: Servers. 
Licenses: All the software used.

Support for licenses and equipment.

Maintenance: for equipment.

We can see that for AYB Host prices can lower with the use of Cloud Azure, but customary costs could differ depending on the use of IT in Azure. In-house IT is more static and with lower occupation it will cost the same. In case of higher occupation, prices will undoubtedly grow more than with the use of Cloud Azure.

\section{Case conclusion}

Cloud Computing enjoys the company's confidence and they are optimistic about it. Trusting this technology is very important for its use. Looking from different perspectives we can detect certain problems, e.g. lack of knowledge. The bad thing about this issue can be insecurity of their partners which will determine lack of trust in this very technology.

Cloud Computing may be helpful for their company. As they use numeral software in developing their applications, Cloud Computing can be very useful and helpful. For their own IT, the Cloud Azure platform that they use for their customers can be a truly beneficial development.

\section{Conclusions}

Larger operations may find switching to the cloud technology somewhat more challenging and as yet less rewarding. Cloud Computing enables businesses and organisations to run faster, more efficient and more flexible IT operations at reduced costs. The calculations provided in the interview section prove the financial advantages, even though the precise degree of flexibility and performance has not been assessed. Based on other sources, however, we can state that these two parameters also speak in favour of the cloud architecture, due to the fact that the Cloud Computing model balances processing power based on the up-to-the-moment demand.

Cloud Computing offers great scalability. As soon as a company is ready to expand, all it needs to do is request more processing power from the provider. At the same time, the client company pays only for the actual capabilities it uses: more for peak processing load times and less for periods of low usage. Reaching a new high in processing power demand does not require purchasing new hardware and all the associated expenses - it rather requires another increase in cloud service usage. Another benefit offered by Cloud Computing, in addition to better performance and flexibility, is reliable and simple backup data storage, physically distributed and so almost invulnerable to damage.

Although the advantages of Cloud Computing more than compensate for the difficulties associated with it, the latter must be carefully considered. As the cloud architecture is relatively new, so far the best guarantee against all risks it entails, from a single company's perspective, is a well-formulated service-level agreement defining 
the terms of service and shared responsibility and security roles between the client and the provider.

\section{Q1: How can a company benefit from using Cloud Computing, compared to} other solutions?

Cloud Computing is a relatively recent addition to the spectrum of available IT technologies, already proving to be more efficacious than traditional IT systems. Most popular types of processing architecture nowadays are either small distributed systems or powerful centralised mainframes. The ever-increasing load of data is straining the capacities of such systems throughout the world, even though currently they still perform quite well. Keeping them powerful enough for carrying out their functions entails periodic upgrades, software enhancements, and other measures, all of which cost money. The ultimate drawback of both traditional architecture types is the processing power reserve: most of the time such systems use only up to onefifth of their total power, and peak loads are rare. Thus, the most significant expenses go toward securing the capabilities used the least, and even these expenses do not guarantee enough power for a new, higher-than-ever load.

This is where Cloud Computing, with its on-demand flexibility, becomes truly useful. The processing capacity of a Cloud Computing system can be increased on demand, and this one-time increase translates into a one-time, exact payment to the provider. As for the upper limit of processing power, a Cloud Computing system has practically none, thanks to the vast distributed network of processors available on demand. This architecture packs more punch for the processing needs of a single IT company than any huge mainframe, while being able to balance multiple loads at a much lower cost to the provider than the older systems.

\section{Q2: What are possible issues that occur with Cloud Computing?}

As any new technology, Cloud Computing has certain issues that must be given due consideration. Most of them are related to privacy and legal aspects, since information is essentially transferred from a company to the cloud and back. As the data is processed within the cloud, the cloud service provider shares the responsibility for keeping all sensitive information secure and safe. The roles of each party at establishing and maintaining information security must then be well-defined and clearly stated in legally binding terms (such as SLA), and security measures must be implemented on both sides working in close cooperation.

Even as a single business may have the security of its information compromised, this is doubly true of a cloud provider. The cloud network with its vast amounts of sensitive information flowing in many directions is an attractive target for cyberattacks, such as denial of service, injection, and other exploits. The nature of Cloud Computing makes it less susceptible to some types of attacks and more to others, but in any case the cloud provider and the company must take all possible steps to reduce network vulnerabilities.

Another important consideration is the sheer size of the cloud network. In most cases, the data is hosted in many countries, which means that the laws of each country are applicable to the data processed over the network, possibly conflicting with the terms of the service-level agreement between the cloud provider and its client. 


\section{Q3: How would Cloud Computing change an organisations' IT infrastructure?}

Other types of IT architecture used by a single business or organisation include distributed networks (in-house), mainframes (in-house) and remote servers. For inhouse computing, upgrades and regular maintenance is performed directly on the premises, and all downtime instances, not to mention outright failures, significantly and immediately affect the company's operations. Remote servers somewhat resemble Cloud Computing in that all maintenance is taken care of by the provider, and the effect of a maintenance procedure in progress upon the business is often cushioned by the provider's server redundancy. However, remote servers have the same drawbacks as mainframe in terms of high costs and the lack of power flexibility.

As organisations and businesses switch to Cloud Computing, all or most processing must occur outside. To be sure, the switch itself might be a demanding, expensive and sometimes lengthy procedure - especially for those who switch from the in-house infrastructure. The companies that already outsource their IT will most probably feel no difference, except for reduced costs and greater processing capabilities available just when they are needed.

Eventually, however, the advantages of Cloud Computing will pay out even for the former in-house IT users.

\section{References}

Armbrust, M. et al. (2009), 'Above the Clouds: A Berkeley View of Cloud Computing', available at: http://www.eecs.berkeley.edu/ Pubs/TechRpts/2009/EECS-2009-28.pdf, Access on March 2014.

Armenian information and communications technology sector 2012 state of industry report (2013). Available at http:// mineconomy.am/uploades/2013210612 3005548.pdf, accessed in March 2014.

Cloud Computing Risk Assessment (2009). European Network and Information Security Agency (ENISA). Available at http://www.enisa.europa.eu/activities/ risk-management/files/deliverables/cloudcomputing-risk-assessment/at_download/ fullReport, accessed in March 2014.

Harutyunyan, A., \& Grigoryan, A. (2010). Development of Resource Sharing System Components for AliEn Grid Infrastructure (No. CERN-THESIS-2010-084). State Engineering University of Armenia.
Harutyunyan, A., Sanchez, C. A., Blomer, J., \& Buncic, P. (2011, December). CernVM Co-Pilot: A framework for orchestrating virtual machines running applications of LHC experiments on the cloud. In Journal of Physics: Conference Series (Vol. 331, No. 6). IOP Publishing.

Johnston, S. (2013). 'Diagram showing overview of cloud computing including Google, Salesforce, Amazon, Axios Systems, Microsoft, Yahoo \& Zoho'. Diagram available on Creative Commons AttributionShare Alike 3.0 at http://en.wikipedia.org/ wiki/File:Cloud_computing.svg\#filelinks accessed in March 2014.

KPMG (2012), Australian Information Industry Association, 'Modeling the economic impact of Cloud Computing', available at http://www.kpmg.com/AU/en/ IssuesAndInsights/ArticlesPublications/ Documents/modelling-economic-impactcloud-computing.pdf. Accessed in March 2014. 
Liu, F. et al (2011). „NIST Cloud Computing Reference Architecture”. National Institute of Standards and Technology of the U.S. Department of Commerce, 'Special Publication 500-292', Available at http:// www.disa.mil/Services/DoD-CloudBroker/ /media/Files/DISA/Services/ Cloud-Broker/nist-cloud-ref-architecture. pdf, Accessed in March 2014.

Microsoft (2010), 'The Economics of the Cloud' available at http://www.microsoft. com/en-us/news/presskits/cloud/docs/theeconomics-of-the-cloud.pdf, accessed in March 2014.
Mohammad, H. (2012). Cloud Computing Uncovered: A Research Landscape. Elsevier Press. pp. 41-85.

Xu, X., \& Zhong, M. (2014). Wireless Body Sensor Networks with Cloud Computing Capability for Pervasive Healthcare: Research Directions and Possible Solutions. In Frontier and Future Development of Information Technology in Medicine and Education (p. 979-988). Springer Netherlands. 